\section{Robust and artifact-free mounting of tissue samples for atomic force microscopy}

\author{
Joshua T. Morgan ${ }^{1,{ }^{*}}$, Vijay Krishna Raghunathan ${ }^{1,{ }^{*}}$, Sara M. Thomasy ${ }^{1}$, \\ Christopher J. Murphy ${ }^{1,2}$, and Paul Russell ${ }^{1}$ \\ ${ }^{1}$ Department of Surgical and Radiological Sciences, School of Veterinary \\ Medicine, University of California, Davis, One Shields Ave, Davis, CA and \\ ${ }^{2}$ Department of Ophthalmology and Vision Science, School of Medicine, \\ University of California, Davis, One Shields Ave, Davis, CA
}

J.T.M. and V.K.R. contributed equally to this work.

BioTechniques 56:40-42 (January 2014) doi 10.2144/000114126

Keywords: atomic force microscopy; tissue mechanics; cornea; trabecular meshwork; sample preparation

Immobilization of tissue-samples for atomic for microscopy (AFM) is typically done using either semi-dry tissue or by gluing the tissue sample down, both of which can introduce artifacts. Here, we describe the design of a SoftClamping Immobilizing Retainer of Tissue (SCIRT) for consistent and nondestructive immobilization of tissues for AFM analysis. We compare the performance of our SCIRT method with glue-immobilization for two difficult to handle tissue types: human trabecular meshwork (HTM) and rabbit cornea (RC). Our results demonstrate that the SCIRT method has several advantages, including: ( $i$ ) allowing for small sample sizes, (ii) enabling continuous hydration, (iii) eliminating contact with glue or associated solvents, (iv) permitting sample recovery following measurement, and $(v)$ ease of use. In conclusion, the SCIRT method is a simple and effective means of immobilizing soft, hydrated tissue samples consistently and without artifacts.

Analogous to chemical signaling, the biophysical properties of the substratum are capable of potently altering cellular behavior and mechanotransduction in response to therapeutic agents $(1-3)$. To further increase the utility of investigations pertaining to cellular mechanotransduction and consequences of biophysical stimuli, rigorous evaluation of relevant tissue properties is required. Unfortunately, extracting tissue mechanics is made difficult by two properties that differ from those of traditional engineering materials. First, the apparent elastic modulus $\left(E_{a p p}\right)$ of tissue (typically $\sim 10^{2}-10^{4} \mathrm{~Pa}$ ) is several orders of magnitude lower than that of non-biological materials (typically $\left.\sim 10^{7}-10^{11} \mathrm{~Pa}\right)$. Second, the oriented nature of matrix proteins leads to highly anisotropic material properties. This can make techniques such as tensile testing unsuitable when trying to determine local nanoscale properties experienced by cells (4). Additionally, most tissues are hydrated in vivo, and their material properties change dramatically upon drying (5-8).

While atomic force microscopy (AFM) has emerged as the gold standard for assaying tissue properties owing to nanoscale resolution and sensitivity in liquid environments $(4,9)$, accurate AFM measurements require sample immobilization, which frequently means either $(i)$ measuring the sample dry or (ii) gluing the sample using fibrin or cyanoacrylate glue. Both of these methods introduce artifacts $(5,6,10)$. Although artifacts can be minimized by keeping the tissue partially moist, minimizing the amount of glue, or discarding obviously artifactual data, such error control methods are subjective and add substantially to the time and expense of accurately measuring tissue samples. To facilitate easier, more consistent AFM measurements, we have developed a robust methodology for mounting hydrated tissue without the need for direct gluing.

Our new technique had to satisfy five major requirements: First, the tissue had to be immobilized in a dish suitable for AFM. Second, the tissue had to be maintained in a hydrated state to prevent drying artifacts. Third, there could be no contact with chemicals that could potentially leach into the tissue and alter its mechanics. Fourth, as tissue samples, especially those from conventional lab animals such as mice, rats, and rabbits, can be quite small, the method had to be adaptable down to sample sizes of 2 $\times 0.1 \times 0.1 \mathrm{~mm}$. Fifth, the method must be nondestructive and allow the sample to be used for further processing. Our method, which satisfies these requirements, is described below and in Figure 1.

To begin, the sample is placed in the center of an AFM dish coated with a layer of silicone (Sylgard 527; 4 h cure at $65^{\circ} \mathrm{C}$; Dow Corning, Midland, $\mathrm{MI}$ ), which helps maintain tissue positioning. A Soft-Clamping Immobilizing Retainer of Tissue (SCIRT) is made by using a $1.25-4 \mathrm{~mm}$ biopsy punch to create a window in a $13 \mathrm{~mm}$ Thermanox coverslip (ThermoFisher Scientific, Waltham, MA), and small droplets of cyanoacrylate glue are placed around its periphery. Utilizing a dissection microscope (Stemi V6, Carl Zeiss AG, Oberkochen, Germany), the SCIRT is then placed such that the tissue is accessible through the window, allowing for it to be monitored throughout the mounting process; this helps to ensure the sample is not physically stretched or compressed by the SCIRT or comes into contact with the

Method summary:

Here we describe an effective and robust glue-less methodology to immobilize soft, hydrated tissue samples for atomic force microscopy without artifact or tissue-damage. 

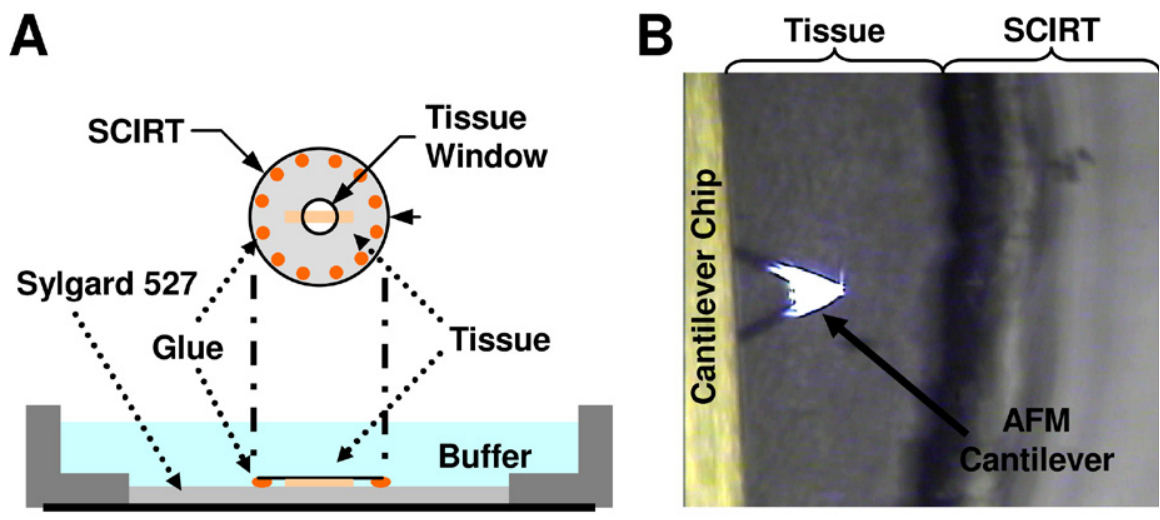

Figure 1. Technique to mount samples using the SCIRT method. (A) Diagram of Soft-Clamping Immobilizing Retainer of Tissue (SCIRT) usage. A $13 \mathrm{~mm}$ Thermanox coverslip is modified using a biopsy punch to allow for a tissue access window. Small $(\sim 0.5 \mathrm{~mm})$ droplets of cyanoacrylate glue are added to the perimeter of the SCIRT using a 22 gauge needle. The SCIRT is placed over the tissue, clamping it into place. The dish is then filled with buffer, curing the glue and keeping the tissue hydrated. (B) Image of rabbit cornea (tissue) held in place using the SCIRT system.

glue. Sample buffer [typically Dulbecco's phosphate buffered saline (DPBS)] is immediately added to the dish to cure the glue and keep the tissue hydrated. The mounting procedure, which includes transfer of the tissue, placement of the SCIRT, and addition of buffer, generally takes an experienced handler less than $30 \mathrm{~s}$; during this time the tissue typically remains wet. If continuous hydration is required, it may be helpful to place a few drops of buffer on the AFM dish during tissue placement. This should not impact SCIRT placement.

To evaluate this method, we chose two previously studied tissue types, rabbit corneal stroma (RC) and human trabecular meshwork (HTM) $(11,12)$. Both tissues are sensitive to hydration. HTM is also an extremely small tissue, with cross-sectional dimensions on the order of $0.1 \mathrm{~mm}$ and sample lengths available for investigation as short as a few millimeters. For each tissue type, we chose six normal donors and split the tissue into two groups. For the first group, the tissue samples were affixed to the dish using an extremely thin layer of glue, as previously described $(11,12)$. For the second group, the tissues were affixed using the method described above (Figure 1), with $4 \mathrm{~mm}$ windows for $\mathrm{RC}$ and $1.5-2 \mathrm{~mm}$ windows for HTM. All human tissue was handled in accordance with the tenets of the Declaration of Helsinki, and all animal tissue was acquired under a protocol approved by the University of California, Davis Institutional Animal Care and Use Committee. Both tissues were carefully isolated from the surrounding tissue as previously described $(11,12)$ and mounted on AFM dishes.

After mounting, force versus indentation curves were obtained as previously described $(11,13,14)$ using an MFP-3D Bio AFM system (Asylum Research, Goleta, CA) and silicon nitride cantilevers (PNP-TR-50, NanoWorld, Neuchâtel, Switzerland) with attached borosilicate microspheres (radius $=4-6 \mu \mathrm{m}$, ThermoFisher Scientific). Each sample was measured at 4-6 locations with 4-7 curves collected at each location. The contact point was determined by a masked analyst and data were fit to a Hertz model (4).

The results of these measurements are shown in Figure 2. It is apparent the SCIRT method yields more consistent median values between samples, although both methods point to an $E_{a p p}$ on the order of $10^{2}-10^{3} \mathrm{~Pa}$, consistent with published results. The difference between the two methods is more striking in the RC samples, with glued samples displaying dramatic intra-sample variability. Also, the SCIRT method results in a far more consistent data range with minimal spread.

Notably, although the glue-immobilized samples can exhibit similar median values compared to samples prepared using our SCIRT method, they also can exhibit a remarkable skew toward stiffer values. Taken together, these data suggest that glue immobilization has the potential to produce artifactually stiff experimental data. While the results presented here only address the elastic properties of the tissue, the method could be readily extended to creep or stress relaxation studies as well.

The mechanism(s) underlying the observed artifactual skew toward stiffness for glue-immobilized tissues is unclear. One likely possibility is glue leaching into the tissue sample. A previous report demonstrated that fibrin glue can increase measured tissue stiffness (10), and it is possible cyanoacrylate glues have a similar effect, which would be exacerbated in thin, porous tissues such as RC and HTM. By avoiding contact between the tissue and glue, the SCIRT method provides a simple and effective way to immobilize hydrated samples for AFM analysis. Importantly, using the SCIRT method, tissue samples remain undamaged after AFM measurements and could therefore be used to isolate RNA/protein or for histology, enabling direct correlation of the mechanical and biological properties of the tissue.
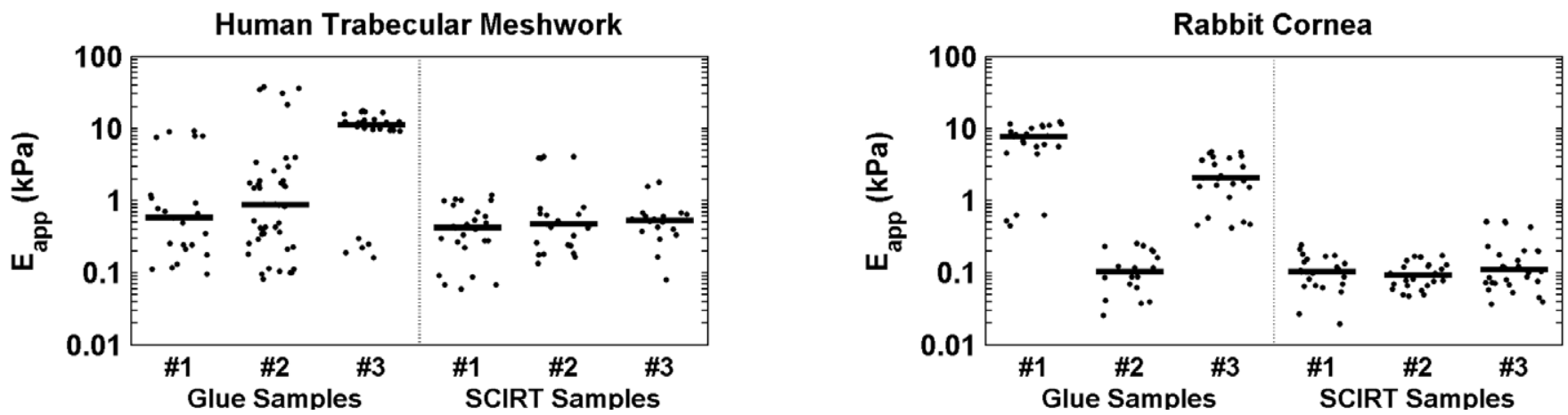

Figure 2. Scatter plot of the force analysis data for HTM (left) and RC (right). The central line indicates the median of the sample. The samples prepared with glue show large variability both within and among the samples for both tissue types. In contrast, the SCIRT immobilized samples had increased consistency of measurement. 


\section{Author contributions}

JTM and VKR designed the study, obtained and analyzed data, and prepared the manuscript. STM did the surgical preparation of cornea tissue and was engaged in editing the manuscript, PR and CJM were involved in study design and manuscript preparation.

\section{Acknowledgments}

We thank Shaun Garland for his technical assistance in this study. This work is supported by grants from the National Institute of Health (R01 EY019475, R01 EY019970 and P30 EY12576) and an unrestricted grant from Research to Prevent Blindness. This paper is subject to the NIH Public Access Policy.

\section{Competing interests}

The authors declare no competing interests.

\section{References}

1. Discher, D.E., P. Janmey, and Y.L. Wang. 2005. Tissue cells feel and respond to the stiffness of their substrate. Science 310:11391143.

2. Yeung, T., P.C. Georges, L.A. Flanagan, B. Marg, M. Ortiz, M. Funaki, N. Zahir, W. Ming, et al. 2005. Effects of substrate stiffness on cell morphology, cytoskeletal structure, and adhesion. Cell Motil. Cytoskeleton 60:24-34

3. Thomasy, S.M., J.A. Wood, P.H. Kass, C.J. Murphy, and P. Russell. 2012. Substratum stiffness and latrunculin $B$ regulate matrix gene and protein expression in human trabecular meshwork cells. Invest. Ophthalmol. Vis. Sci. 53:952-958.

4. McKee, C.T., J.A. Last, P. Russell, and C.J. Murphy. 2011. Indentation versus tensile measurements of Young's modulus for soft biological tissues. Tissue Eng. Part B Rev. 17:155-164.

5. Ebenstein, D.M. and L.A. Pruitt. 2006. Nanoindentation of biological materials. Nano Today 1:26-33.

6. Oyen, M.L. 2011. Nanoindentation of Biological and Biomimetic Materials. Exp. Techniques 37:73-87.

7. Haut, T.L. and R.C. Haut. 1997. The state of tissue hydration determines the strain-ratesensitive stiffness of human patellar tendon. J. Biomech. 30:79-81.

8. Constantinides, G., Z.I. Kalcioglu, M. McFarland, J.F. Smith, and K.J. Van Vliet. 2008. Probing mechanical properties of fully hydrated gels and biological tissues. J. Biomech. 41:3285-3289.

9. Radmacher, M. 1997. Measuring the elastic properties of biological samples with the AFM. IEEE Eng. Med. Biol. Mag. 16:47-57.

10. Chaurasia, S.S., R. Champakalakshmi, A. Li, R. Poh, X.W. Tan, R. Lakshminarayanan, C.T. Lim, D.T. Tan, and J.S. Mehta. 2012. Effect of fibrin glue on the biomechanical properties of human Descemet's membrane. PLOS ONE 7:e37456.

11. Last, J.A., T. Pan, Y. Ding, C.M. Reilly, K. Keller, T.S. Acott, M.P. Fautsch, C.J. Murphy, and P. Russell. 2011. Elastic modulus determination of normal and glaucomatous human trabecular meshwork. Invest. Ophthalmol. Vis. Sci. 52:2147-2152.

12. Thomasy, S.M., V.K. Raghunathan, M. Winkler, C.M. Reilly, A.R. Sadeli, P. Russell, J.V. Jester, and C.J. Murphy. Elastic modulus and collagen organization of the rabbit cornea: epithelium to endothelium. Acta Biomater. (In press).

13. Last, J.A., S.J. Liliensiek, P.F. Nealey, and C.J. Murphy. 2009. Determining the mechanical properties of human corneal basement membranes with atomic force microscopy. J. Struct. Biol. 167:19-24.

14. Last, J.A., S.M. Thomasy, C.R. Croasdale, P. Russell, and C.J. Murphy. 2012. Compliance profile of the human cornea as measured by atomic force microscopy. Micron 43:12931298.

Received 08 October 2013; accepted 12 December 2013.

Address correspondence to Paul Russell, Department of Surgical \& Radiological Sciences, School of Veterinary Medicine, University of California Davis, Davis, CA. E-mail: prussell@ ucdavis.edu

To purchase reprints of this article, contact: biotechniques@fosterprinting.com

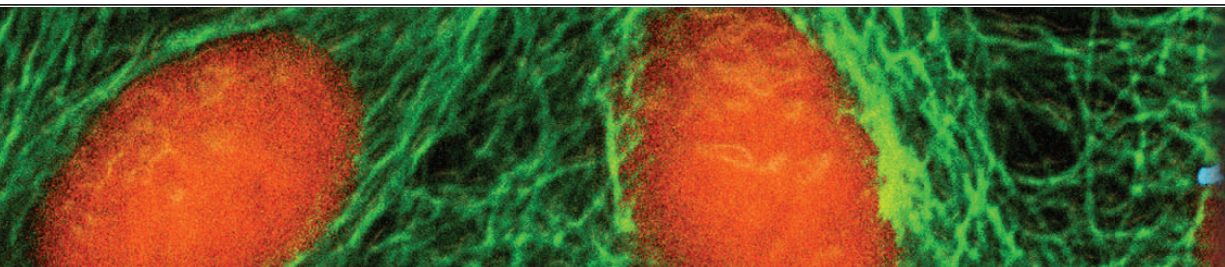

\section{BioTechniques Newsletters}

\section{Your Daily Science Fix}

BioTechniques Newsletters and Alerts complement the print journal by highlighting late-breaking news, feature articles, advanced access to upcoming content, industry events, videos, and more.

Join the more than 80,000 subscribers who receive one or all:

Daily and Weekly Newsletters

Electronic Table of Contents and BenchTalk Podcast Alerts

Technology-specific Newsletters

Cell Analysis/Culture

DNA Sequencing

- Genetic Engineering Tools \& Techniques

- Genomics/Genetics Tools
In Vivo Imaging

PCR/Cloning

Protein Purification \& Analysis
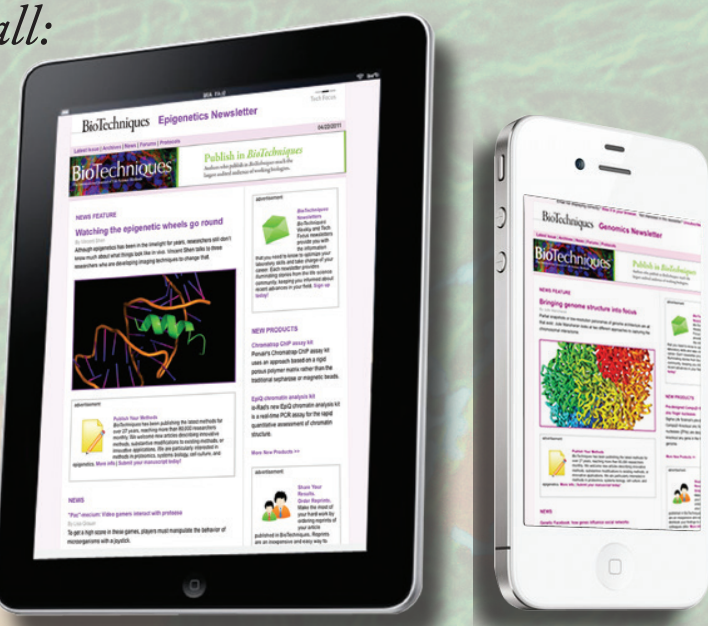